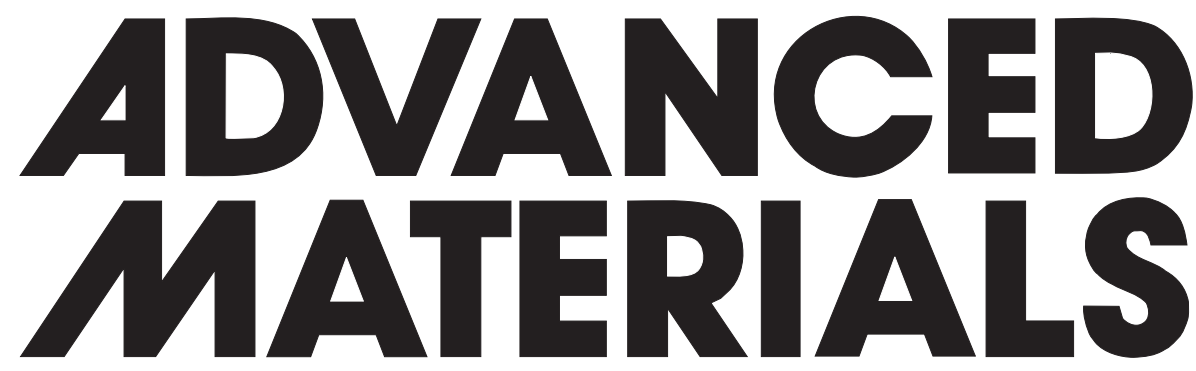

Supporting Information

for

Advanced Materials, adma.200600562

(C) Wiley-VCH 2006

69451 Weinheim, Germany 


\section{One-pot Synthesis of Poly(cyclotriphosphazene-co-4,4'-sulfonyldiphenol) Nanotubes via in situ Template Approach}
$\mathrm{Lu} \mathrm{Zhu}{ }^{1}$, Youyong $\mathrm{Xu}^{3}$, Weizhong Yuan ${ }^{1}$, Jingyu $\mathrm{Xi}^{1}$, Xiaobin Huang ${ }^{1}$, Xiaozhen Tang ${ }^{1,2^{*}}$, Sixun Zheng ${ }^{1 *}$

${ }^{1}$ College of Chemistry and Chemical Engineering, Shanghai Jiao Tong University, 800 Dongchuan Road, Shanghai, 200240, China;

${ }^{2}$ State Key Laboratory of Metal Matrix Composites, Shanghai Jiao Tong University, 1954 Hua Shan Road, Shanghai, 200030, China;

${ }^{3}$ Makromolekulare Chemie II, Universität Bayreuth, D-95440 Bayreuth, Germany

Tel: 86-21-54743264, Fax: 86-21-54741297.

Email:xtang@sjtu.edu.cn (X.Tang),szheng@sjtu.edu.cn (S. Zheng).

1) Materials

2) Measurements

3) Varying Dimension of PZS Nanotubes

4) Explanation of Open and Close Nanotube Ends

5) Experimental Data for Characterization of Chemical Structure of PZS Nanotubes

6) EDX Spectra of In-Process Products

7) Evidence for Complete Removal of TEACI Templates

8) Determination of Contact Angle Values between Probe Liquids and PZS Nanotubes or TEACI Crystals

9) Determination of Adhesion Characteristics of TEACl-filled PZS Core/Shell Composite

10) References

1) Materials 
Hexachlorocyclotriphosphazene (HCCP) (Aldrich) was recrystallized from dry hexane followed by two times sublimation. The melting point of the purified HCCP was $113-114{ }^{\circ} \mathrm{C}$. 4,4'-sulfonyldiphenol and triethylamine were purchased from Shanghai Chemical Reagents Corp. (Shanghai, China) and used without further purification. Tetrahydrofuran (THF) was distilled from $\mathrm{CaH}_{2}$ under dry $\mathrm{N}_{2}$ purge. Hexane were distilled from $\mathrm{P}_{2} \mathrm{O}_{5}$.

\section{2) Measurements}

The fourier transform infrared spectroscopy (FTIR) measurements were conducted on a Perkin-Elmer Paragon 1000 Fourier transform spectrometer at room temperature $\left(25^{\circ} \mathrm{C}\right)$. The samples were mixed with the powder of $\mathrm{KBr}$ and then pressed into the flakes. The specimens were sufficiently thin to be within a range where the Beer-Lambert law is obeyed. In all cases, 64 scans at a resolution of $2 \mathrm{~cm}^{-1}$ were used to record the spectra. All magic angle spinning (MAS) solid state nuclear magnetic resonance (NMR) spectra were acquired using a Varian Mercury plus 400 spectrometer. The resonance frequencies of ${ }^{13} \mathrm{C}$ and ${ }^{31} \mathrm{P}$ were 100.63 and $161.99 \mathrm{MHz}$, respectively. The powder samples were loaded into $4 \mathrm{~mm}$ diameter zirconia MAS rotors and spun at the magic angle at rates of 3 and $6 \mathrm{kHz}$, respectively, and all measurements were done at room temperature. The ${ }^{13} \mathrm{C}$ cross polarization (CP) / MAS NMR spectrum was recorded using a $4.0 \mathrm{~s}$ recycle delay, $2.5 \mathrm{~ms}$ contact time, and about $56 \mathrm{kHz} \mathrm{rf}$ fields for both cross polarization and high-power dipolar decoupling (DD). ${ }^{13} \mathrm{C}$ chemical shifts relative to tetramethylsilane $\left(\mathrm{Me}_{4} \mathrm{Si}\right)$ were determined by using glycine as an external reference. Quantitative ${ }^{31} \mathrm{P}$ MAS NMR spectra were recorded by a one-pulse sequence with the high-power DD technique. The proton $\pi / 2$ pulse duration was $5 \mu$ s and 2000 signal transients with a 100 s relaxation delay were accumulated. Magic-angle spinning was set at 6 $\mathrm{kHz}$ in order to ensure complete separation of sideband intensity from the central transition. Ammonium dihydrogen phosphate (ADHP) was used as a ${ }^{31} \mathrm{P}$ chemical shift reference $(\delta=$ $0.8 \mathrm{ppm})^{[1]}$ and as an external reference. Elemental analyses were performed on a Perkin 
Elmer 2400-II element analyzer. X-ray diffraction (XRD) patterns were recorded by using a Bruker D8 Advance instrument equipped with $\mathrm{Cu} \mathrm{K} \alpha$ radiation performed at $40 \mathrm{kV}$ and 40 $\mathrm{mA}$. The scan range was $1.0^{\circ} / \mathrm{min}$ from $5^{\circ}$ to $60^{\circ}(2 \theta)$. Field emission scanning electron microscope (FE-SEM) images were obtained using a Philips Sirion 200 instrument at an activation voltage of $20 \mathrm{kV}$. All specimens were obtained by depositing the tetrahydrofuran suspension on glass substrates and allowed to dry in air under a lamp. The thin films were then coated with thin layers of gold of about $100 \AA$. Transmission electron microscopy (TEM) images were obtained using JOEL JEM-100CX instrument operated at $100 \mathrm{kV}$ equipped with an energy-dispersive X-ray analyzer (Genesis spectrum, EDXA, Inc.). The specimens were obtained from aspirating the tetrahydrofuran suspensions on carbon-coated copper grids and allowed to dry in air under a lamp. High resolution transmission electron micrographs (HR-TEM) were recorded on a Philips CM200 FEG microscope operating at $120 \mathrm{kV}$.

\section{3) Varying Dimension of PZS Nanotubes}

If the molar ratio of HCCP to BPS was set to be $1: 2$, TEACl crystals were precipitated out while polymer was soluble instead of precipitating out due possibly to the low degree of crosslinking. Consequently, the crystals grew thicker without being covered by the polymer. Within ten minutes, supplemental BPS was added to attain the molar ratio of HCCP to BPS of $1: 3$, and then polymers were immediately precipitated out due to the occurrence of crosslinking. With the polymers adhering onto the nanocrystals, the growth of the nanocrystals in the direction of radii was suppressed whereas the crystals can still grow along the direction of length. The TEM results indicate that the tubes with inner diameter of 10-20 $\mathrm{nm}$ were obtained (Figure $2 \mathrm{f}, \mathrm{g}$ ) and most tips of the tubes are still open (Figure $2 \mathrm{~h}$ ). It is noted that the lengths of the tubes are quite dependent on the lengths of the crystals. As the power 
of the ultrasonic irradiation was increased, the crisp TEACl crystals were broken up to shorter rods; consequently, lengths of the tubes were set within $1 \sim 2 \mu \mathrm{m}$ (Figure $2 \mathrm{i}$ ).

\section{4) Explanation of Open and Close Nanotube Ends}

It should be pointed out that majority of the obtained tubes ends are open, however, when the ultrasonic irradiation with higher power was exerted on the reactive system, the most of the tube ends are closed as shown in Figure 2i. This observation could be interpreted on the basis of the competitive kinetics between growth of TEACl nanocrystals and absorption of polymer particles on the surfaces of TEACl nanocrystals. If the rate of the former is higher than that of the latter, the open ends of the nanotubes will be obtained. To confirm this, we re-observed the formation of the nanotubes by means of TEM and we indeed observed the case that the length increase of TEACl nanocrystal is faster than the absorption of the polymer particles on the surface of the nanocrystal (See Figure S4 d \& e). It should be pointed out that long TEACl nanocrystals can be broken into short nanorods under ultrasonic irradiation of increased power. In this case, the rate of length increase for a single TEACl crystalline nanorod was decreased and thus more closed ends can be formed (See Figure 2i) since the rate of absorption of polymer particles on the surfaces of TEACl nanocrystal remains invariant.

\section{5) Experimental Data for Characterization of Chemical Structure of PZS Nanotubes}

The result of fourier transform infrared (FTIR) spectroscopy demonstrates the presence of phosphazene, sulfone, phenylene and $\mathrm{P}-\mathrm{O}-\mathrm{C}(\mathrm{Ph})$ groups in $\mathrm{PZS}$ nanotubes (Figure S1). ${ }^{13} \mathrm{C} \mathrm{CP} / \mathrm{MAS}$ NMR and quantitative ${ }^{31} \mathrm{P}$ MAS NMR spectra of PZS nanotubes are employed to characterize the polymer (Figure S2). ${ }^{13} \mathrm{C}$ NMR spectrum shows phenylene groups' peak at 153, 139, 129 and $121 \mathrm{ppm}$. In the ${ }^{13} \mathrm{C} \mathrm{CP} / \mathrm{MAS}$ NMR spectrum, no resonance of carbon at $161 \mathrm{ppm}$ was detected, suggesting that all phenolic hydroxyl groups of

BPS had completely reacted with HCCP. In the quantitative ${ }^{31} \mathrm{P}$ NMR spectrum of PZS 
nanotubes, two resonance signals appeared at 3 and $19 \mathrm{ppm}$, respectively, indicating the presence of the structural units of $-\mathrm{N}=\mathrm{P}\left(-(\mathrm{OPh})_{2}\right)-$ and $-\mathrm{N}=\mathrm{P}(-\mathrm{OPh})(-\mathrm{Cl})-$. The ratio of integration intensity at the two resonances shows that about one third of phosphorus atoms exist in the form of $-\mathrm{N}=\mathrm{P}(-\mathrm{OPh})(-\mathrm{Cl})-$ in the crosslinked $\mathrm{PZS}$, which was further confirmed by elemental analyses. On the basis of the above analysis, the molecular structure is presented in Figure S2 as highly cross-linked networks with assignments of ${ }^{13} \mathrm{C}$ NMR and ${ }^{31} \mathrm{P}$ NMR.

Anal. Calcd. for $\mathrm{C}_{30} \mathrm{H}_{22} \mathrm{ClN}_{3} \mathrm{O}_{10} \mathrm{P}_{3} \mathrm{~S}_{2.5}$ (PZS): C, 45.4; H, 2.8; Cl, 4.5; N, 5.3; P, 11.7; S, 10.1. Found: C, 43.2; H, 2.8; Cl, 6.1; N, 5.2; P, 11.7; S, 10.1. 


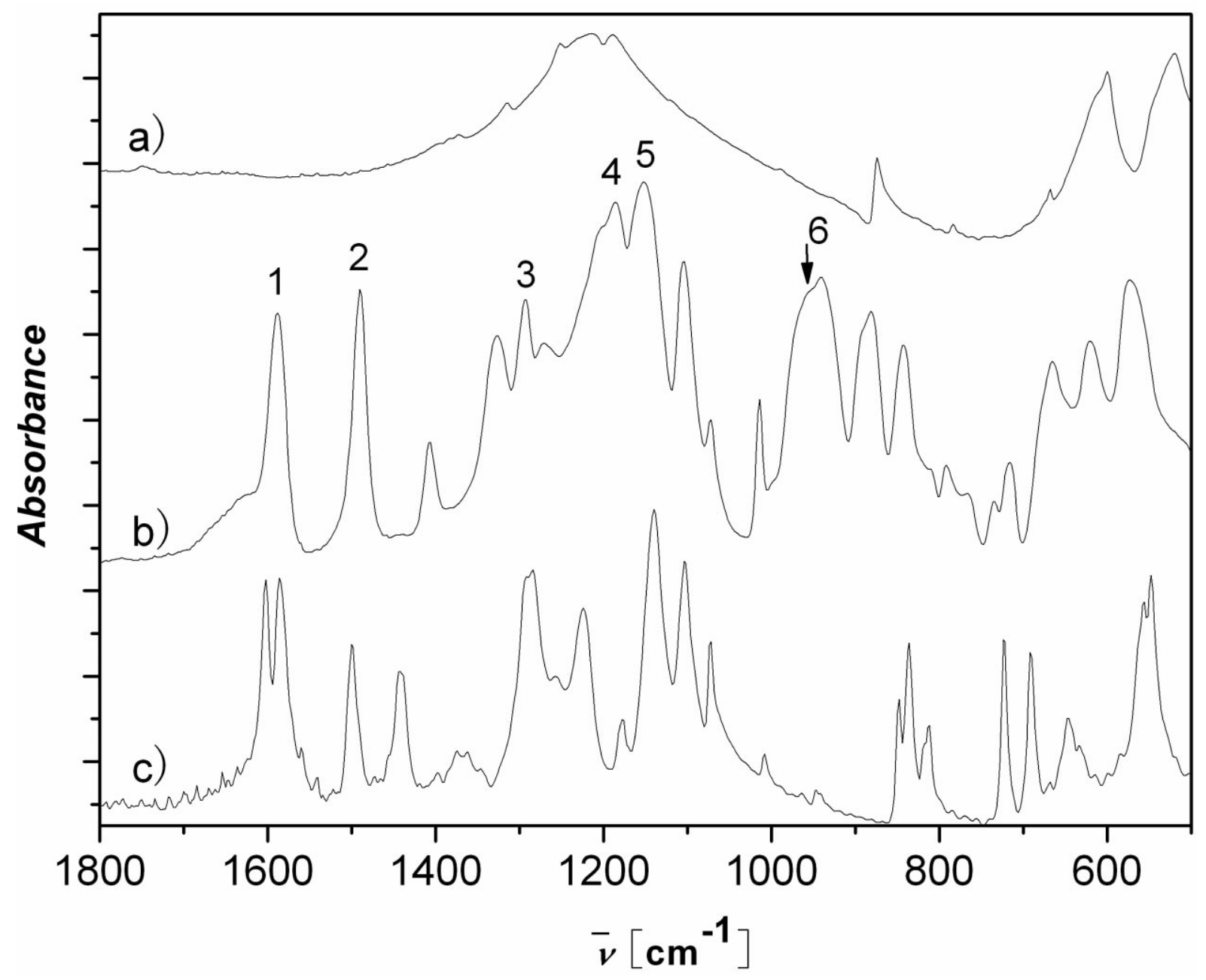

Figure S1. IR spectrum of the monomers and PZS nanotubes: a) hexachlorocyclophosphazene, b) PZS nanotubes and c) 4,4'-sulfonyldiphenol. Assignment of spectrum of PZS nanotubes: peak 1 and 2: $\mathrm{C}=\mathrm{C}(\mathrm{Ph})$ str, peak 3 and 5: $\mathrm{O}=\mathrm{S}=\mathrm{O}$ str, peak 4: $\mathrm{P}=\mathrm{N}$ valence, peak 6: $\mathrm{P}-\mathrm{O}-(\mathrm{Ph})$ str. 


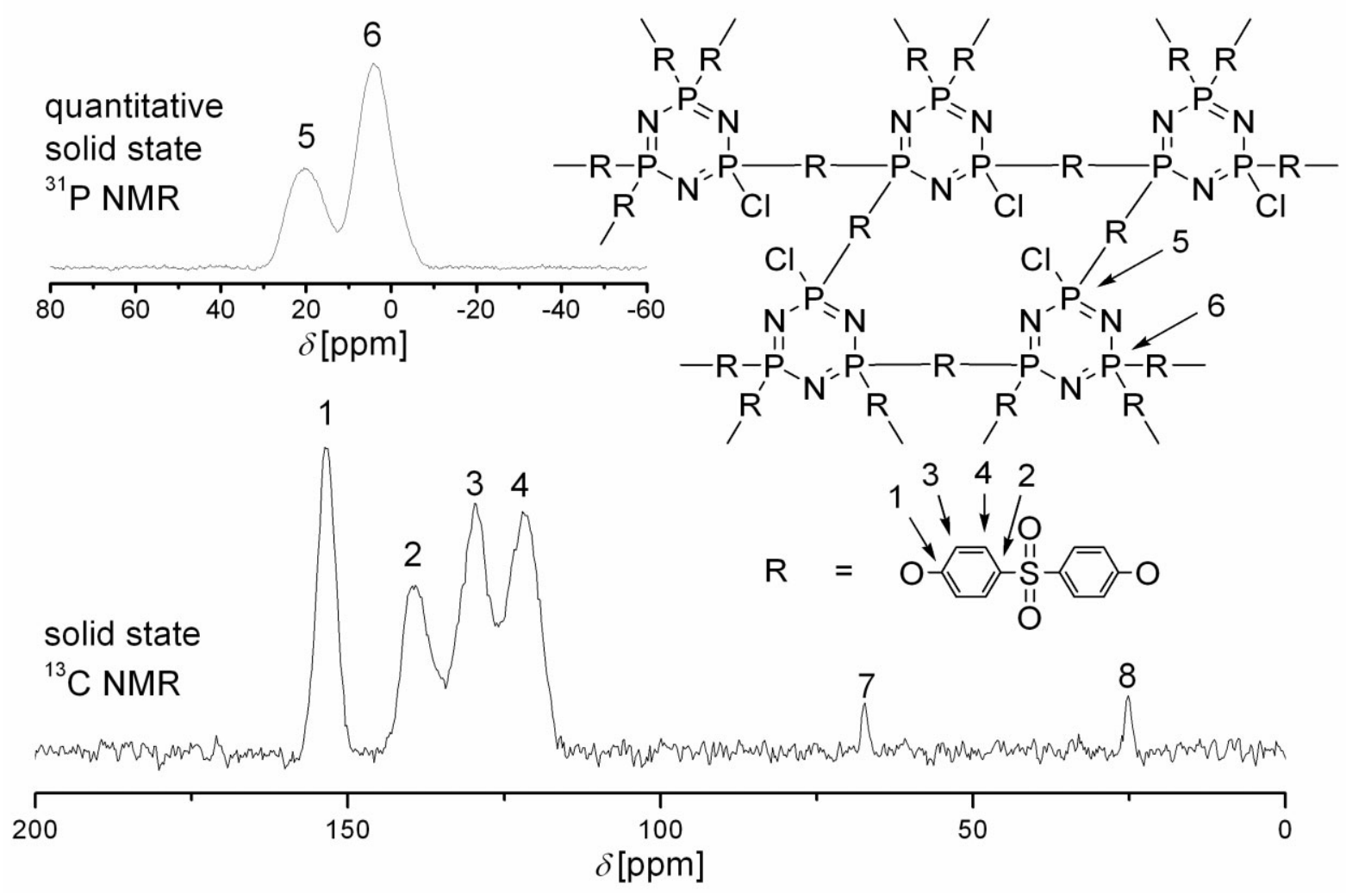

Figure S2. Solid state ${ }^{13} \mathrm{C}$ and quantitative solid state ${ }^{31} \mathrm{P}$ NMR spectra of PZS nanotubes and highly cross-linked molecular structure of PZS with assignments of ${ }^{13} \mathrm{C}$ and ${ }^{31} \mathrm{P}$ NMR spectra. (Peaks 7 and 8 in ${ }^{13} \mathrm{C}$ NMR spectrum are attributed to tetrahydrofuran absorbed into the tubes) 


\section{6) EDX Spectra of In-Process Products}

EDX analysis of the in-process products shows that the spherical nanoobjects possess the identical composition with PZS nanotubes whereas the nanorods are composed of carbon and chlorine.
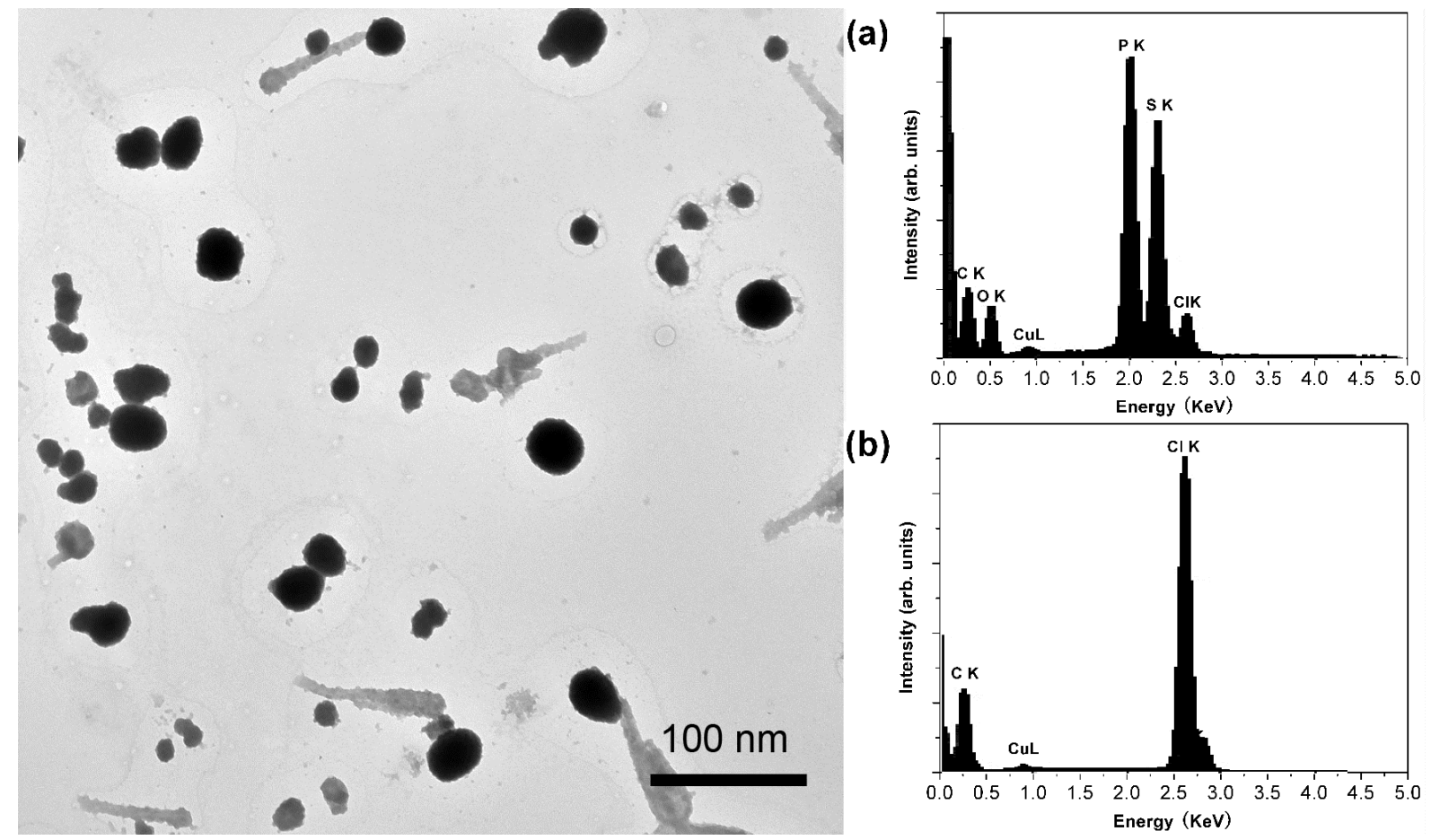

(b)

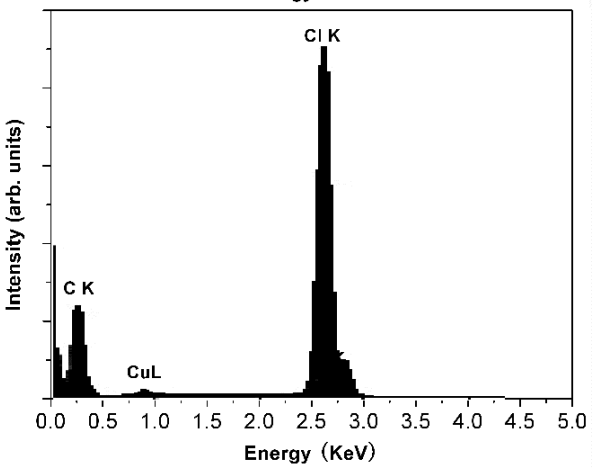

Figure S3. Results of EDX analysis of the nano-spheres (dark) and nano-rods (grey) in TEM images of the in-process products (left), a) and b) respectively. 


\section{7) Evidence for Complete Removal of TEACI Templates}

As shown in Figure S4 a, b and c, the crystals of TEACl appeared as bright (a), invisible (b) or dark (c) rods when the specimens were prepared with different thickness. So, one can not confirm the existence of crystals in the PZS nanotubes only by the TEM images.

The EDX spectrum in Figure S4f displayed a signal at $\mathrm{E}=2.62 \mathrm{keV}$ ascribable to $\mathrm{Cl}$ $\mathrm{K} \alpha$, indicating the vast presence of $\mathrm{Cl}$ element in the nanocomposites. However, the above mentioned signals distinctly reduced in Figure S4i. The disappearance of the $\mathrm{Cl}$ content after the nanocomposites were washed by water supports the view that the crystals of TEACl were removed by water. The existence of a small quantity of chlorine in the nanotubes was caused by the unreacted $\mathrm{P}-\mathrm{Cl}$ bonds in the polymer. Both Figure S4f and i show signals of copper, carbon, oxygen, phosphorus and sulfur originated from the copper grid and PZS. We have also taken the electron diffraction (ED) pattern for the selected area shown in Figure S4e and $h$ (inserts of Figure $\mathrm{S} 4 \mathrm{e}$ and $\mathrm{h}$, respectively). In Figure $\mathrm{S} 4 \mathrm{e}$, the diffraction feature of the patterns is manifesting the presence of TEACl nanocrystals; in Figure S4h, the very weak diffuse ring of ED patterns indicates the amorphous structure of the selected area containing no crystalline, which shows that the core template in the cylinder has been removed away.

Although the completely disappearance of the crystals can not be ascertained from the TEM images or EDX spectra, the FT-IR (Figure S5), NMR (Figure S6) and XRD (Figure S7) data of a sample of the products before and after washed by water do confirm the completely removal of the crystals of TEACl:

FTIR As shown in Figure S5, the characteristic absorption of triethylammonium could be located at 2976, 2939, 2678 and $2491 \mathrm{~cm}^{-1}$ in the FTIR spectra of both TEACl and the nanocomposites, as shown in the figure. From the total disappearance of the above absorptions due to triethylammonium groups in the IR spectrum of the nanotubes, it can be concluded that the removal of the TEACl templates is complete; 
NMR As shown in Figure S6, strong resonances of the alkyl carbon of TEACl were detected at 47.5 and 9.5 ppm before the sample was washed by water. However, those two peaks did no longer exist at all after the sample was washed by water, which proved the complete removal of the core templates;

XRD As shown in Figure S7, the sharp reflections of the nanocomposites can be indexed to hexagonal TEACl. However, in the measurements of the sample washed by water, no sharp diffraction peaks but wide diffuse scattering peaks were observed. This is another evidence supporting the completely removal of the templates of TEACl crystals.

This observation is in marked contrast to that reported by Yang et al. ${ }^{[2,3]}$ for the penetration of water into a nanoscale hollow cylinder with $10 \mathrm{~nm}$ inner diameters by diffusion. In the present case, the complete removal of TEACl could be due to the high solubility of TEACl in water. 

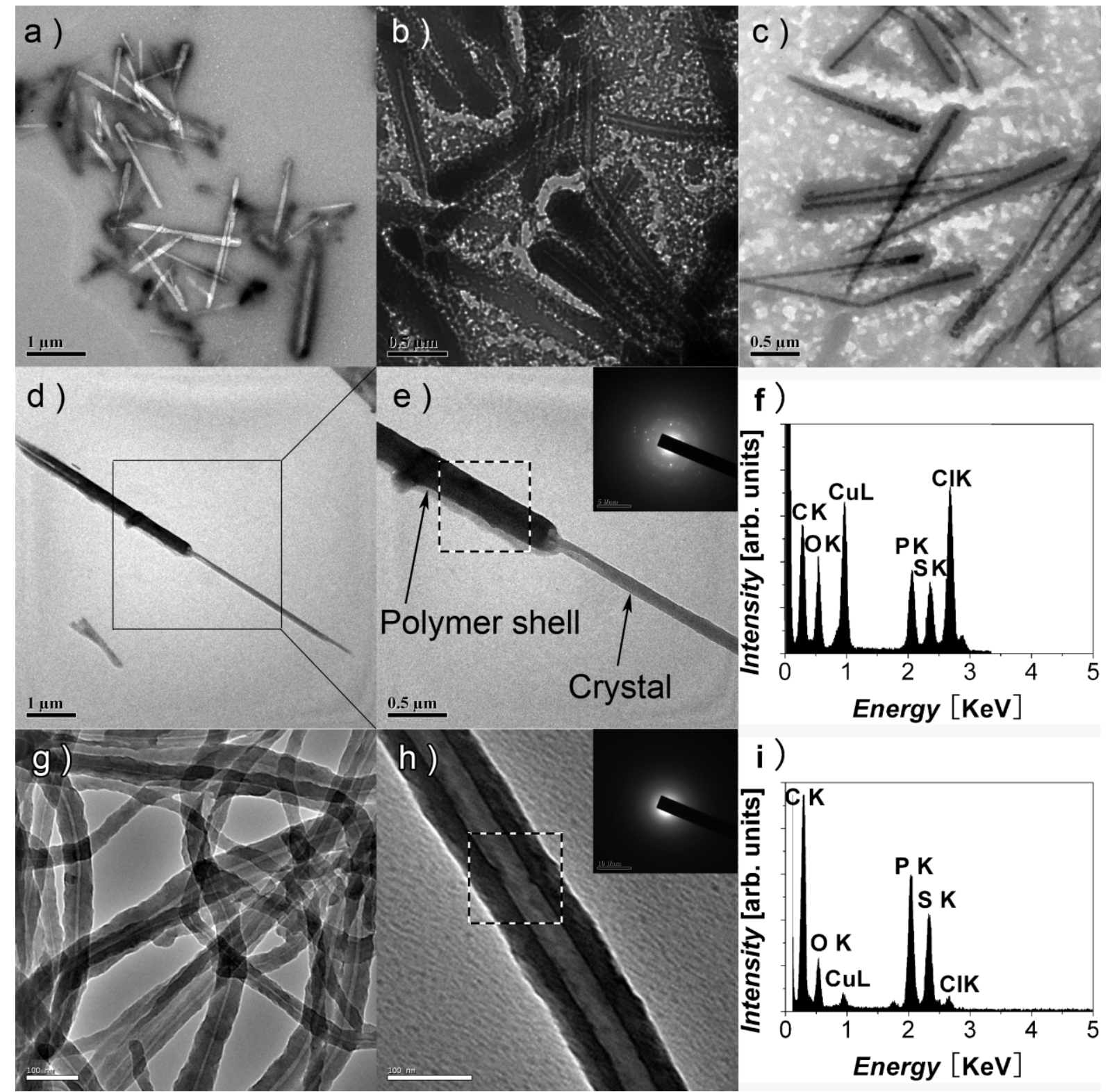

Figure S4. a, b, c, d, e) TEM images obtained from samples of the PZS-TEACl nanocomposites, insert of e) is the electron diffraction (ED) pattern of the selected area; $g, h$ ) TEM images of the PZS nanotubes without core templates(ie. after washing by water), insert of h) is the ED pattern of the selected area; f, i) EDX spectra of the selected area in e) and h), respectively. Scale bar: a, d) $1 \mu \mathrm{m}$; b, c, e) $0.5 \mu \mathrm{m}$; g, h) $100 \mathrm{~nm}$. 


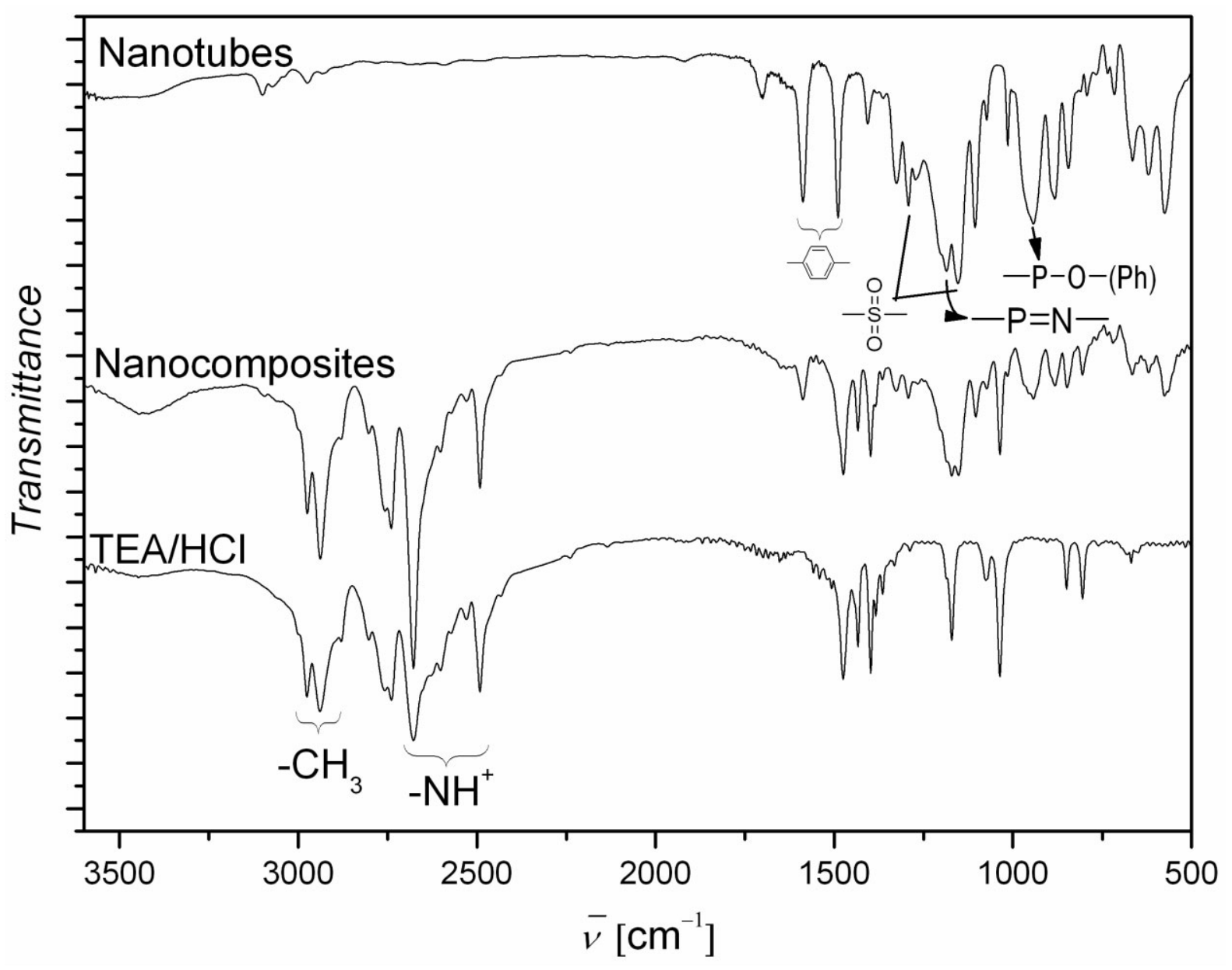

Figure S5. IR spectra of the PZS-TEACl nanocomposite, the PZS nanotubes without core templates (ie. a sample before and after washing by water) and crystals of TEACl. The characteristic absorption of triethylammonium could be located at 2976, 2939, 2678 and 2491 $\mathrm{cm}^{-1}$ in the FTIR spectra of both TEACl and the nanocomposites, as shown in the figure. From the total disappearance of the above absorptions due to triethylammonium groups in the IR spectrum of the nanotubes, it can be concluded that the removal of the TEACl templates is complete. 


$$
\left.\mathrm{Cl}^{-}+\mathrm{HN}+\underset{1}{+}+\underset{2}{\mathrm{C}}-\mathrm{CH}_{3}\right)_{3}
$$
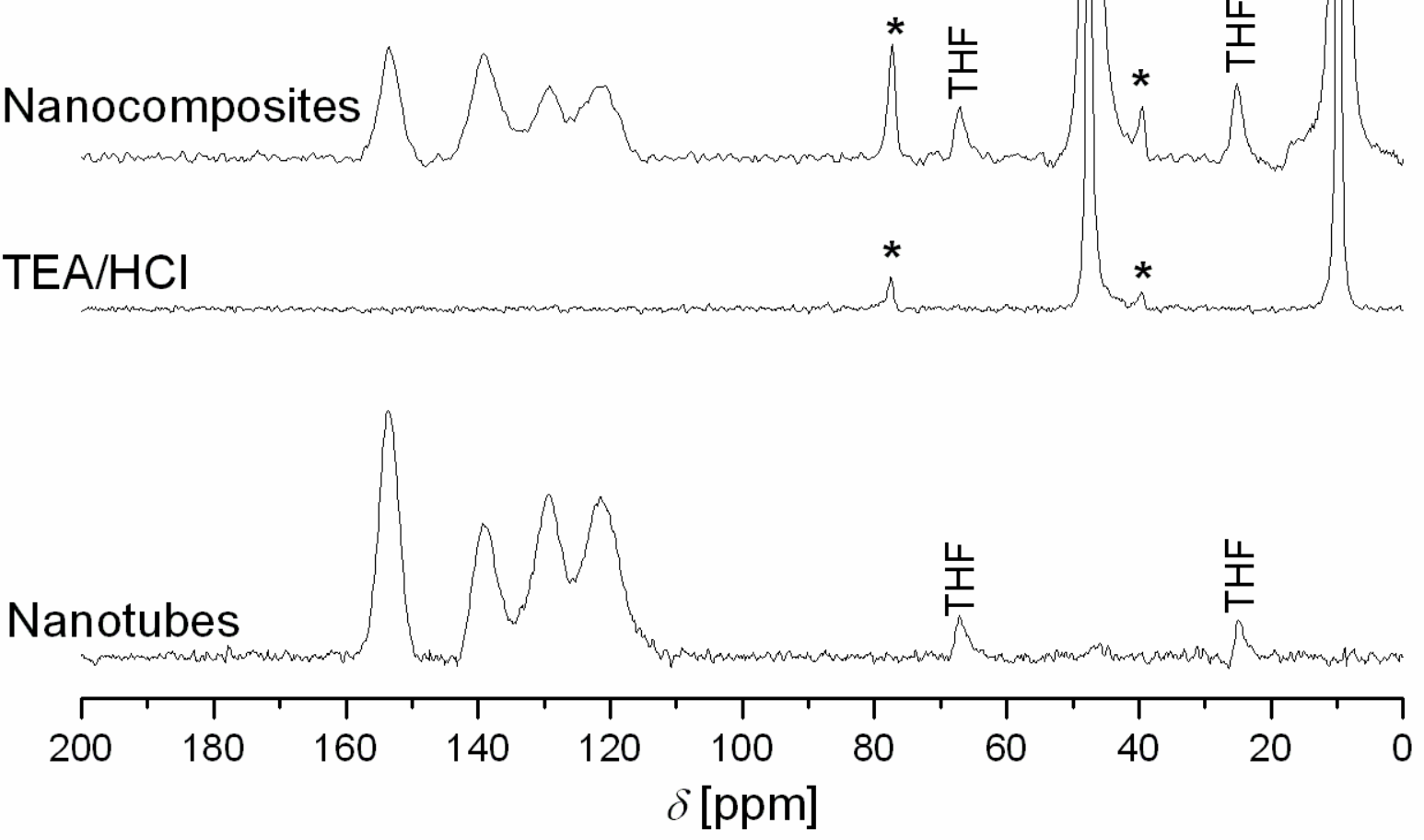

Figure S6. Solid state ${ }^{13} \mathrm{C}$ NMR spectra obtained from TEACl, the PZS-TEACl nanocomposites and the PZS nanotubes without core templates. As shown in the figure, strong resonances of the alkyl carbon of TEACl were detected at 47.5 and $9.5 \mathrm{ppm}$ before the sample was washed by water. However, those two peaks did no longer exist after the sample was washed by water. ( $*$ : Spinning sidebands) 


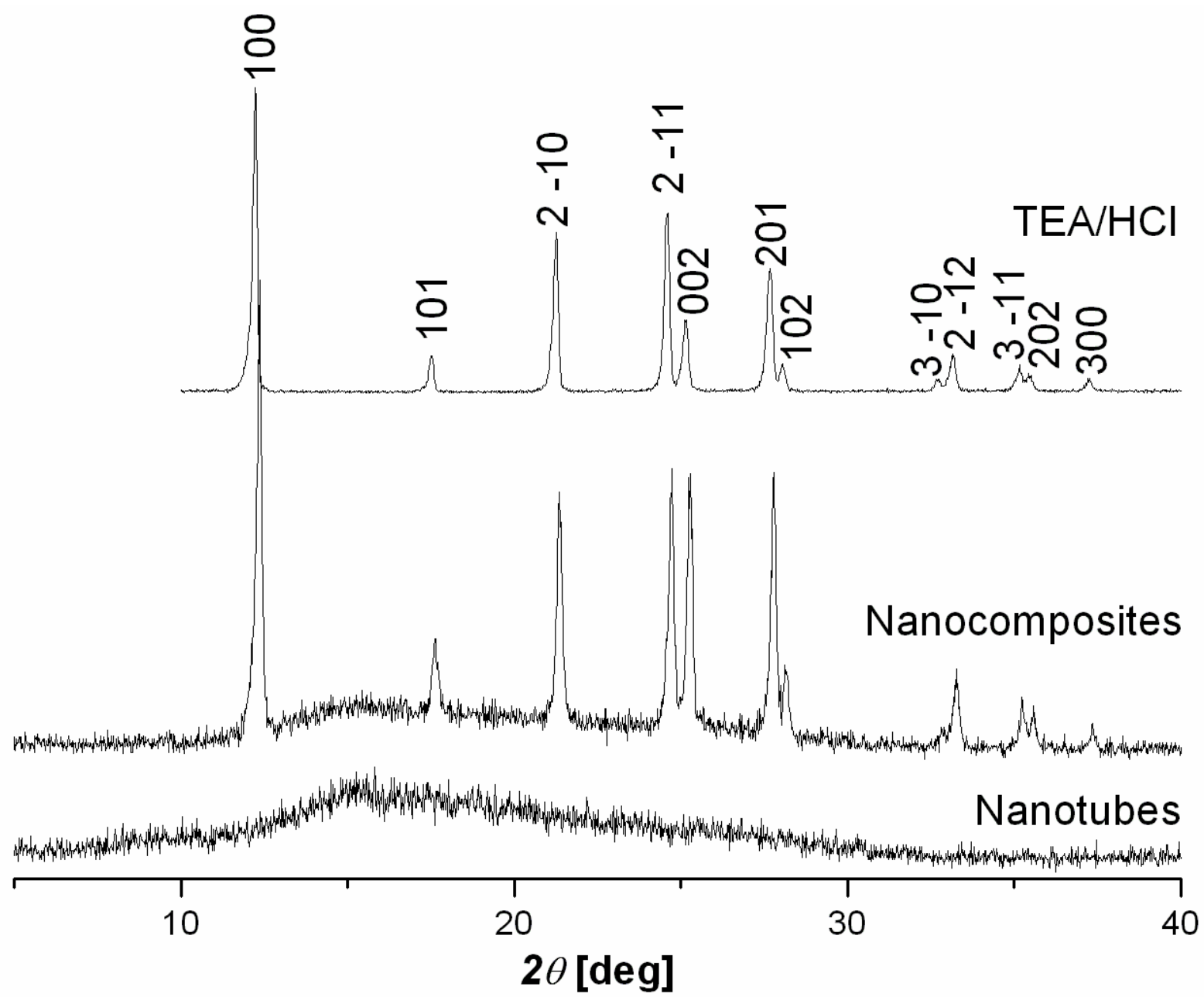

Figure S7. XRD pattern recorded from TEACl, the PZS-TEACl nanocomposites and the PZS nanotubes without core templates. The reflections disappeared after wash can be indexed to hexagonal TEACl with a lattice constant of $\mathrm{a}=8.340 \AA$, consistent with the standard value (JCPDS file 38-1974, $\mathrm{a}=8.342 \AA$ ) within experimental error, which further confirms that the clever templates is TEACl. Furthermore, in order to obtain better contrast, the XRD pattern for the actually obtained crystals of TEACl is also given in the figure. It is noted that the obtained TEACl crystals are several microns in diameters owing to the difficulty in collecting nano-crystals of TEACl from the reaction media. 


\section{8) Determination of Contact Angle Values between Probe Liquids and PZS Nanotubes or TEACl Crystals}

Contact angle values between liquids and powdered TEACl are determined with the thin-layer wicking technique. ${ }^{[4]}$

$$
x^{2}=\frac{R t}{2 \eta} \gamma_{1} \cos \theta
$$

Contact angle values between liquids and porous powdered PZS nanotubes are determined with special modified thin-layer wicking technique developed by Cui et al. ${ }^{[5]}$

$$
\begin{aligned}
& \qquad \frac{d x^{2}}{d t}=\frac{R_{P} K}{2 \eta} \gamma \cos \theta \\
& \text { where }=\frac{V_{P}}{\left(V_{b}-V_{a d s}\right)}
\end{aligned}
$$

The thin-layer wicking experiments were performed using glass plates $(25 \times 80 \mathrm{~mm})$ on which a suspension of each powdered material was deposited. The suspension was prepared with $0.5 \mathrm{~g}$ of PZS nanotubes or $2.5 \mathrm{~g}$ TEACl in $50 \mathrm{ml}$ of petroleum ether and $5 \mathrm{ml}$ portions were pipetted onto each plate placed horizontally to obtain a uniform layer. After petroleum ether evaporation ( 1 or 2 hours), the plates were dried at $100{ }^{\circ} \mathrm{C}$ for $2 \mathrm{~h}$ and kept in a desiccator. The plates were allowed to equilibrate in a closed vessel with the saturated vapor of each probe liquid for $24 \mathrm{~h}$. The probe liquids were $n$-hexane, $\alpha$-bromonaphthaline and diiodomethane. Polar liquids such as aniline, formamide and water were excluded because of solubility of TEACl and swelling of PZS in them. All experiments were carried out at a room temperature of $20-22{ }^{\circ} \mathrm{C}$.

For PZS nanotubes plates, the volume of liquid adsorbed $\left(V_{a d s}\right)$ is calculated from the difference in weight before and after pre-contact. By weighing the plates at the end of the wicking experiments, it is possible to calculate the total volume of the pore space $\left(V_{b}\right)$. The maximum volume of capillary condensed liquid $\left(V_{\max }\right)$ was assumed to be the same as that for 
$n$-hexane determined gravimetrically in the same way by putting the plates in the desiccator for sufficient time for complete equilibration with the saturated vapor. The volume of the through channels $\left(V_{p}=V_{b}-V_{\max }\right)$ can be calculated and hence $K$ determined from equation 3 . The radius of the through channels $\left(R_{p}\right)$ was determined first using $n$-hexane with presaturated plates. The plates were then thoroughly dried out in an oven at $100{ }^{\circ} \mathrm{C}$ and stored in a desiccator until used. Wicking experiments were then carried out with the other probe liquids on both bare and pre-contacted plates. The results are summarized in Table S1 (Figure S8) and Table S2 in which equation 2 has been used to calculate contact angle. It was assumed that bare plates give advancing angles and pre-contacted ones give receding angles, regardless of the extent of the preadsorption by capillary condensation. ${ }^{[5]}$ A notable feature of the contact angle results for the probe liquids, listed in Table S3, is the small difference between advancing and receding angles. This gives confidence in the calculation of an "equilibrium" contact angle $\left(\theta_{0}\right)$ using the equation 4.

$$
\cos \theta_{0}=\left(\cos \theta_{a}+\cos \theta r\right) / 2
$$

For TEACl plates, pre-contact experiment showed that adsorptive capacity of hexane is very small (value of $V_{\max } / V_{b}$ was less than 5\%), so TEACl plate doesn't have blind pores. Wicking of probe liquids into TEACl obeyed to Washburn Equation (equation 1). Wicking curves of bare and pre-contact plates are almost the same one. So, the calculated out $\theta$ could be considered as "equilibrium" contact angle $\theta_{0}$, listed in Table S3. 
Table S1. Wicking of probe liquids into TEACl and PZS nanotubes

$$
T / \mathrm{s}
$$

\begin{tabular}{|c|c|c|c|c|c|c|c|c|c|}
\hline \multirow{3}{*}{$\begin{array}{l}X / \\
\mathrm{cm}\end{array}$} & \multicolumn{6}{|c|}{ Polymer } & \multirow{2}{*}{\multicolumn{3}{|c|}{ TEACl }} \\
\hline & \multicolumn{3}{|c|}{ Bare } & \multicolumn{3}{|c|}{ Pre-contact } & & & \\
\hline & Hexane & $\begin{array}{c}\alpha- \\
\mathrm{BrC}_{10} \mathrm{H}_{7}\end{array}$ & $\mathrm{CH}_{2} \mathrm{I}_{2}$ & Hexane & $\alpha-\mathrm{BrC}_{10} \mathrm{H}_{7}$ & $\mathrm{CH}_{2} \mathrm{I}_{2}$ & Hexane & $\begin{array}{c}\alpha- \\
\mathrm{BrC}_{10} \mathrm{H}_{7}\end{array}$ & $\mathrm{CH}_{2} \mathrm{I}_{2}$ \\
\hline 0 & 0 & 0 & 0 & 0 & 0 & 0 & 0 & 0 & 0 \\
\hline 0.5 & 7 & 21 & 15 & 1 & 24 & 43 & 2 & 4 & 4 \\
\hline 1 & 11 & 117 & 83 & 6 & 86 & 64 & 6 & 29 & 9 \\
\hline 1.5 & 29 & 233 & 226 & 19 & 245 & 150 & 19 & 98 & 37 \\
\hline 2 & 50 & 442 & 323 & 33 & 453 & 279 & 34 & 166 & 81 \\
\hline 2.5 & 79 & 679 & 534 & 52 & 698 & 451 & 53 & 266 & 157 \\
\hline 3 & 122 & 966 & 759 & 78 & 955 & 580 & 73 & 396 & 218 \\
\hline 3.5 & 151 & 1346 & 1097 & 107 & 1298 & 816 & 99 & 546 & 312 \\
\hline 4 & 194 & 1725 & 1428 & 141 & 1591 & 1053 & 121 & 717 & 427 \\
\hline 4.5 & 237 & 2143 & 1721 & 179 & 2032 & 1332 & 155 & 914 & 584 \\
\hline 5 & 294 & 2787 & 2210 & 228 & 2436 & 1719 & 185 & 1160 & 750 \\
\hline 5.5 & 361 & 3498 & 2879 & 273 & 2926 & 2127 & 223 & 1391 & 970 \\
\hline 6 & 441 & 4182 & 3495 & 331 & 3415 & 2535 & 260 & 1686 & 1194 \\
\hline 6.5 & 502 & 4758 & 3954 & 398 & 4089 & 2900 & 311 & 2028 & 1441 \\
\hline 7 & 574 & 5333 & 4472 & 459 & 4652 & 3287 & 354 & 2340 & 1737 \\
\hline
\end{tabular}




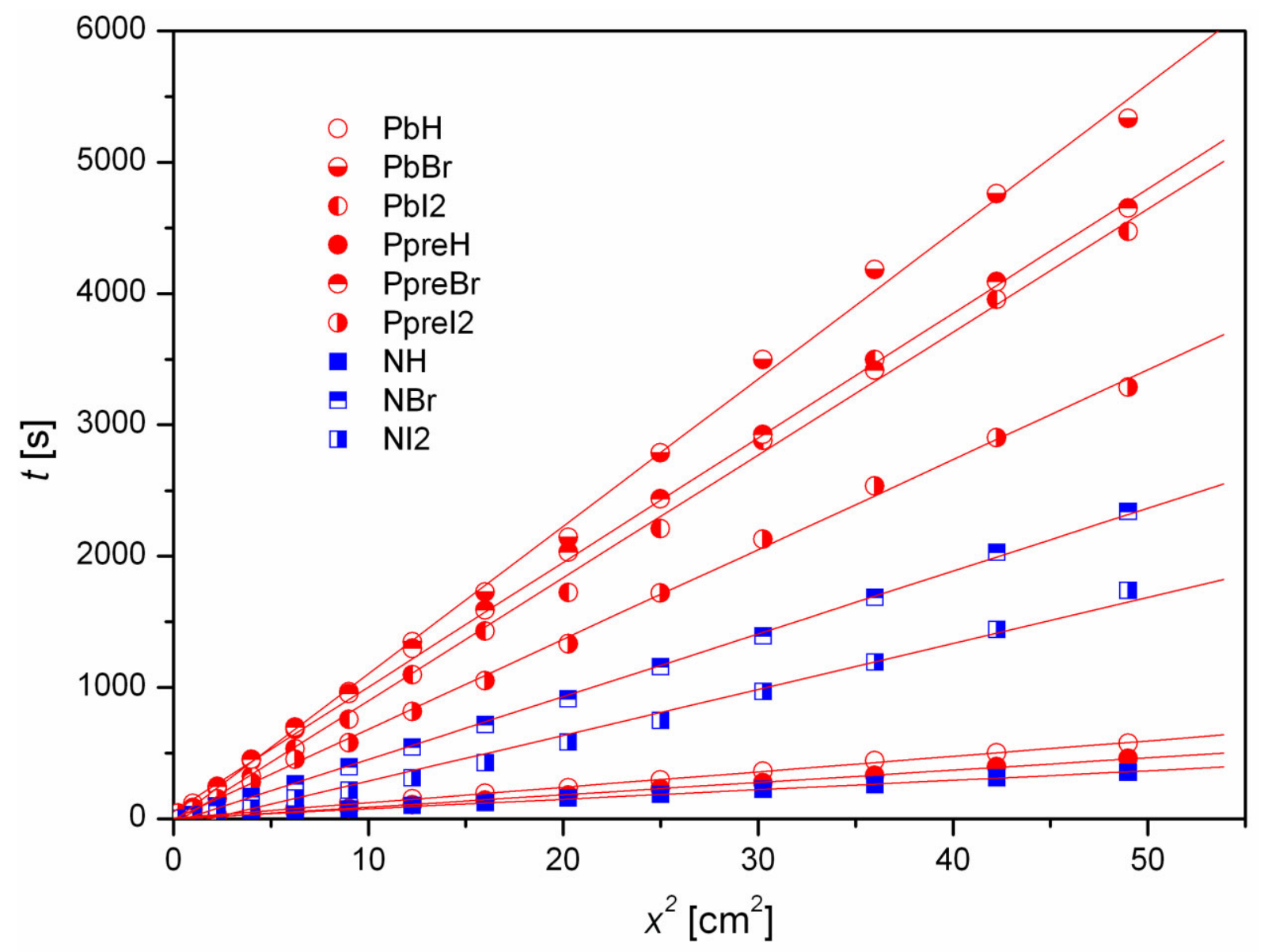

Figure S8. Wicking of probe liquids into TEACl and PZS nanotubes. (PbH: n-Hexane into bare PZS plates; $\mathrm{PbBr}$ : $\alpha$-bromonaphthalene into bare $\mathrm{PZS}$ plates; $\mathrm{PbI}_{2}$ : diiodomethane into bare PZS plates; PpreH: n-Hexane into precontact PZS plates; PpreBr: $\alpha$-bromonaphthalene into precontact PZS plates; $\mathrm{PpreI}_{2}$ : diiodomethane into precontact PZS plates; NH: n-Hexane into TEACl plates; $\mathrm{NBr}$ : $\alpha$-bromonaphthalene into TEACl plates; $\mathrm{NI}_{2}$ : diiodomethane into TEACl plates. 
Table S2. Wicking of probe liquids into TEACl and PZS nanotubes

\begin{tabular}{|c|c|c|c|c|c|c|c|}
\hline & & $V_{a d s} / \mathrm{cm}^{3}$ & $V_{p} / \mathrm{cm}^{3}$ & $V_{b} / \mathrm{cm}^{3}$ & $K$ & $\left(d x^{2} / d t\right) / \mathrm{cm}^{2} \mathrm{~s}^{-1}$ & $R_{P} / \mu \mathrm{m}$ \\
\hline \multicolumn{8}{|c|}{ PZS nanotubes } \\
\hline \multirow{2}{*}{$n$-Hexane } & Bare & 0 & & 0.3473 & 0.7950 & 0.08482 & 0.3774 \\
\hline & $\begin{array}{c}\text { Pre- } \\
\text { contact }\end{array}$ & 0.0712 & 0.2761 & & 1 & 0.1066 & 0.3771 \\
\hline \multirow{2}{*}{$\alpha-\mathrm{BrC}_{10} \mathrm{H}_{7}$} & Bare & 0 & & & 0.7950 & 0.008915 & \\
\hline & $\begin{array}{l}\text { Pre- } \\
\text { contact }\end{array}$ & 0.0452 & & & 0.9139 & 0.01054 & \\
\hline \multirow{2}{*}{$\mathrm{CH}_{2} \mathrm{I}_{2}$} & Bare & 0 & & & 0.7950 & 0.01068 & \\
\hline & $\begin{array}{c}\text { Pre- } \\
\text { contact }\end{array}$ & 0.0646 & & & 0.9766 & 0.01462 & \\
\hline \multicolumn{8}{|c|}{ TEACl } \\
\hline$n$-Hexane & & & & & & 0.1385 & 0.4900 \\
\hline$\alpha-\mathrm{BrC}_{10} \mathrm{H}_{7}$ & & & & & & 0.02092 & \\
\hline $\mathrm{CH}_{2} \mathrm{I}_{2}$ & & & & & & 0.02860 & \\
\hline
\end{tabular}

Table S3. Contact angle values between hexane, $\alpha$-bromonaphthalene, diiodomethane and triethylamine hydrochloride, PZS nanotubes (in degree)

\begin{tabular}{ccccc}
\hline & & $n$-Hexane & $\alpha$-bromonaphthalene & diiodomethane \\
\hline & $\theta_{a}$ & 0 & 51.1 & 67.9 \\
PZS nanotubes & $\theta_{r}$ & 0 & 49.8 & 65.2 \\
& $\theta_{0}$ & 0 & 50.4 & 66.6 \\
TEACl & $\theta_{0}$ & 0 & 25.6 & 51.9 \\
\hline
\end{tabular}




\section{9) Determination of Adhesion Characteristics of TEACl-filled PZS Core/Shell Composite}

TEACl in a matrix of PZS will either be spontaneously wetted or not by the polymer, depending on the relative intensity of the two surface energy components $\left(\gamma_{S V}\right.$ and $\left.\gamma_{L V}\right)$ and their interactions. Lee has developed an analytical method (unified theory for adhesion, Table S4) for determining their properties. ${ }^{[6]}$ From the contact angle values and employing the unified theory for adhesion, solid/vapor surface energy $\left(\gamma_{S V}\right)$ and interaction parameter $(b)$ are calculated (Table S5). From the $\gamma_{S V}$ and $b$ value, Harkin's spreading coefficient $\left(\lambda_{L S}\right)$ and the adhesion work $\left(W_{S L}\right)$ are calculated (Table S6). Based on the adhesion theory, criteria for predicting the adhesion characteristics of multicomponent polymer systems were established by plotting $\left(\gamma_{S V} / \gamma_{L V}\right)$ vs. ${ }^{\bar{b}}{ }^{[6]}$ For TEACl-filled PZS core/shell composite, the plot of $\gamma_{S V} / \gamma_{L V}$ vs. $\bar{b}$ are showing in Figure S9. It can be concluded that TEACl crystals are wetted by PZS very well. Adhesion energy is high, thus caused the good stability of the core/shell structure even under ultrasonic bath. 
Table S4. Summary of the relationship derived from the unified theory for adhesion ${ }^{[6]}$

Equation of state

$\cos \theta=1+b \ln \frac{\gamma_{C}}{\gamma_{L V}}$

Solid/vapor surface energy

$\gamma_{S V}=\left[b \exp \left(\frac{1}{b}-1\right)\right] \gamma_{C}$

Liquid/solid surface energy

$\gamma_{L S}=\gamma_{S V}+\gamma_{L V}-\gamma_{L V}\left[1+b \exp \left(1-\frac{1}{b}\right)+b \exp \left(1-\frac{1}{b}\right) \ln \left(\frac{\gamma_{S V}}{b \gamma_{L V}}\right)\right]$

Equilibrium work of adhesion

$W_{A}=\gamma_{L V}\left(2+b \ln \frac{\gamma_{C}}{\gamma_{L V}}\right)$

Harkins' spreading coefficient

$\lambda_{L S}=\gamma_{S V} b \exp \left(1-\frac{1}{b}\right)\left(1+\ln \frac{\gamma_{S V}}{b \gamma_{L V}}\right)-1$ 
Table S5. Solid / Vapor surface energy $\gamma_{\mathrm{sv}}$ and interaction parameter for TEACl and PZS nanotubes

\begin{tabular}{ccc}
\hline & $\gamma_{s v} \mathrm{~mJ} / \mathrm{m}^{2}$ & $b$ \\
\hline PZS nanotubes & 41.46 & 1.78 \\
TEACl & 52.99 & 2.12 \\
\hline
\end{tabular}

Table S6. $\bar{b}, \gamma_{S V} / \gamma_{L V}, \lambda_{L S}$ and $W_{S L}$ values for TEACl filler PZS

\begin{tabular}{cccc}
\hline $\bar{b}$ & $\gamma_{S V} / \gamma_{L V}$ & $\lambda_{L S} \mathrm{~mJ} / \mathrm{m}^{2}$ & $W_{S L} \mathrm{~mJ} / \mathrm{m}^{2}$ \\
\hline 1.94 & 1.278 & 96.24 & 88.33 \\
\hline
\end{tabular}




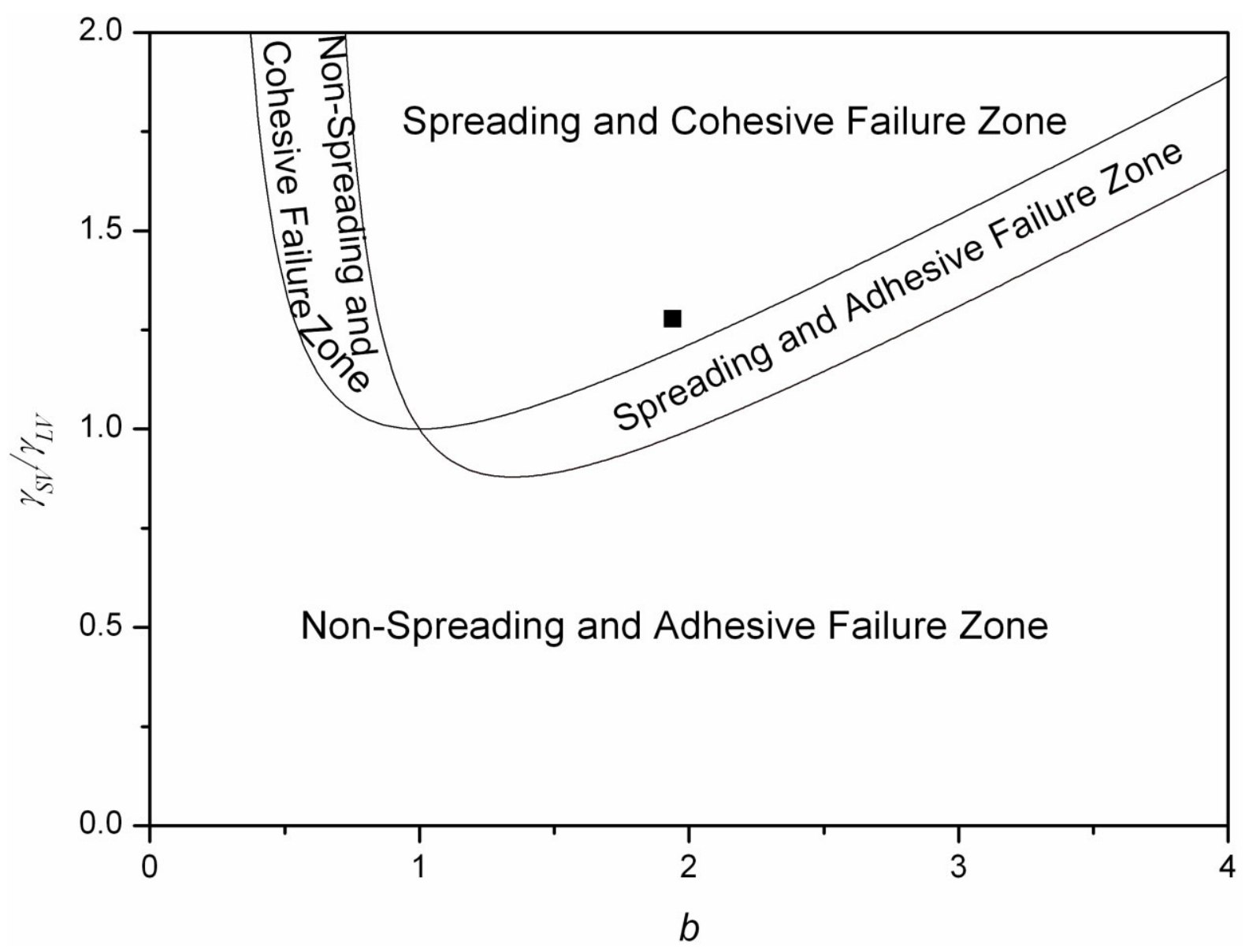

Figure S9. Criteria for predicting Spreading and failure characteristics of multicomponent polymer systems based on the unified theory for adhesion. Square: plot of $\gamma_{\mathrm{SV}} / \gamma_{\mathrm{LV}}$ vs. $\bar{b}_{\mathrm{O}}$ TEACl-filled PZS core/shell composite. 


\section{0) References}

[1] S. Hayashi, K. Hayamizu, Bull. Chem. Soc. Jpn. 1989, 62, 2429.

[2] B. Yang, S. Kamiya, K. Yoshida, T. Shimizu, Chem. Commun. 2004, 500.

[3] B. Yang, S. Kamiya, Y. Shimizu, N. Koshizaki, T. Shimizu, Chem. Mater. 2004, 16, 2826.

[4] E. Chibowski, F. González-Caballero, Langmuir 1993, 9, 330.

[5] Z. G. Cui, B. P. Binks, J. H. Clint, Langmuir 2005, 21, 8319.

[6] M. C. H. Lee, Adhesive Chemistry Development and Trends, Lee, L. H., Ed., Plenum, New York, 1985, 93. 